\title{
Numerical simulation of GLARE 4A fiber-metal laminates subjected to ballistic impact
}

\author{
George Bikakis ${ }^{1,2 *}$, Nikolaos Tsigkros ${ }^{2}$, Emilios Sideridis ${ }^{2}$, and Alexander Savaidis ${ }^{1}$ \\ ${ }^{1}$ School of Pedagogical and Technological Education, Mechanical Engineering Educators \\ Department, GR 14121 N. Heraklion, Athens, Greece \\ ${ }^{2}$ National Technical University of Athens, Strength of Materials Laboratory, GR 15773 Zographou, \\ Athens, Greece
}

\begin{abstract}
This article deals with the evaluation of the ballistic resistance of GLARE 4A fiber-metal laminates subjected to high velocity impact by a cylindrical projectile. Important impact variables such as the ballistic limit, the impact load and the absorbed energy time histories are predicted using the ANSYS LS-DYNA software. The simultaneous existence of various impact damage mechanisms, which is unique in fiber-metal laminates, is demonstrated using the numerical results. Each of the mechanisms absorbs a part of the initial impact energy and contributes to the high ballistic impact resistance the materials. With reference to the considered GLARE 4A panels, the behavior of the transient impact load is analyzed and useful conclusions are drawn. It is found that the maximum impact load is applied at the beginning of ballistic impacts, during the initial local indentation of the panels under the projectile. It is substantially higher than the following peak values of the impact force time history. It is revealed that during the beginning of ballistic impacts, the impulse of the collision increases as the thickness of the panels is increased. The work done by the impact load during the local indentation stage is also an increasing function of the panels' thickness.
\end{abstract}

\section{Introduction}

Fiber-metal laminates are hybrid composite materials, consisting of alternating metal layers bonded to fiber-reinforced prepreg layers. GLARE is the most successful fiber-metal laminate up to now and is currently being used for the construction of primary aerospace structures, such as the fuselage of the Airbus A380 air plane. Further applications have also been considered: aircraft cargo floors of Boeing 777, aircraft engine cowlings, bonded GLARE patch repair, aircraft stiffeners with a wide variety of shapes, cargo containers, seamless GLARE tubes.

Impact properties are very important in aerospace structures, since impact damage is caused by various sources, such as maintenance damage from dropped tools, collision between service cars or cargo and the structure, bird strikes, and hail. For this reason, many researchers have studied the response of composite and GLARE structures to impact

\footnotetext{
* Corresponding author: bikakis.george@,yahoo.com
} 
loading. In pertinent impact studies, the static indentation, the low and high velocity impact, and the ballistic impact response of GLARE are treated using analytical, numerical, and experimental methods.

This article deals with the response of square clamped GLARE 4A fiber-metal laminated plates subjected to central normal ballistic impact by a rigid free-flying cylindrical projectile. GLARE 4A is one of the commercially available grades of GLARE with a specific layup, described in section 3 of this article. The principal objective of the article is to assess the ballistic resistance of GLARE 4A fiber-metal laminates.

In order to simulate the ballistic impacts, ANSYS LS-DYNA software is employed. Important impact variables such as the ballistic limit and the transient impact load are predicted. The presented results will help engineers and researchers, occupied with similar theoretical or experimental impact studies of GLARE hybrid laminates, to understand the considered physical phenomenon and to classify GLARE 4A material grade as far as its ballistic resistance is concerned. To the authors' knowledge, the high velocity ballistic resistance of GLARE 4A material grade has not been studied previously in other scientific publications.

\section{Finite element modeling}

In order to simulate the normal ballistic impacts of free-flying projectiles on GLARE 4A targets, we use the structural arrangement of the ballistic impact experiments of reference [1]. In our finite element modeling procedure we employ SOLID164 elements for the meshing of the projectile and the targets. Two solid elements are used along the thickness of metal layers and one solid element is used along the thickness of glass-epoxy layers of the fiber-metal laminates. The same mesh density along the thickness of fiber-metal laminates is employed in references [2,3].

In order to simulate the contact between the projectile and the panel we use the surfaceto-surface contact type with the eroding contact option. For the simulation of the contact between adjacent layers of the fiber-metal laminate we use the surface-to-surface contact type with the tiebreak contact option. The tiebreak contact option is needed in order to allow delamination/debonding between adjacent layers. Details concerning the simulation of delamination/debonding can be found in reference [2].

In order to reduce the computational cost, the projectile is considered rigid. For this reason, a rigid material model is used for the projectile. The simplified Johnson-Cook plasticity material model [4] is employed for modeling the material behavior of aluminum. We idealize the material behavior of the unidirectional glass-epoxy layers in the fiber-metal laminates by employing the Chang-Chang composite failure model [4].

For the simulation of the perforation of the panels, the panels' finite elements in way of the projectile must be eroded. In order to achieve this goal, a strain-based erosion criterion is added to the aluminum and glass-epoxy material models.

In order to further reduce the high computational cost of the considered problem, we take advantage of the symmetry of the problem by modeling a quarter of the structure and by applying suitable symmetry boundary conditions to all nodes of the symmetry planes.

Given that the projectile is considered rigid, instead of meshing a solid cylinder, only the exterior surface with $0.8 \mathrm{~mm}$ thickness was meshed. The density of the projectile's material was modified so that the full projectile's mass remains constant. A fine mesh is used for the projectile in order to represent its geometry accurately, including the radius of its impacting face. With this meshing approach the number of the finite elements needed to model the projectile is minimized and the computational cost is further reduced. In order to verify the accuracy of the hollow projectile model FEM results, complete projectile models were also implemented and solved yielding practically identical results. 
The one-point integration has been selected for the SOLID164 elements. For each ballistic impact case we analyze, we verify that the hourglass energy and the sliding energy are small relative to the internal energy, as recommended for an explicit analysis with LSDYNA [5].

An explicit transient dynamic analysis is employed with geometric and material nonlinearities. The initial velocity of the projectile is predetermined. The duration of our analysis is controlled with a predetermined termination time which allows for complete panel perforation, provided that the initial velocity of the projectile is greater than or equal to the ballistic limit. It is noted that in order to determine the unknown ballistic limit of a specific panel, several trial analyses have to be executed with different initial projectile velocity.

In order to verify the convergence of FEM results, the ballistic limit of the panel, we built two models with increasing in-plane mesh density for each specific case of square panel we analyze. The projectile's mesh density remains the same for all models. For each model we obtain the ballistic limit and compare them in order to verify that satisfactory convergence has been achieved. A fine mesh model of a GLARE 4A-6/5-0.5 fiber-metal laminate along with the projectile is depicted in Figure 1. In this figure, each layer of the laminate has a different color.

It is noted that the implemented finite element modeling procedure has also been used in reference [2] in order to predict the ballistic impact response of fiber-metal laminates and monolithic metal plates consisting of different aluminum alloys, and by comparison with published experimental data the validity of the procedure was demonstrated there.
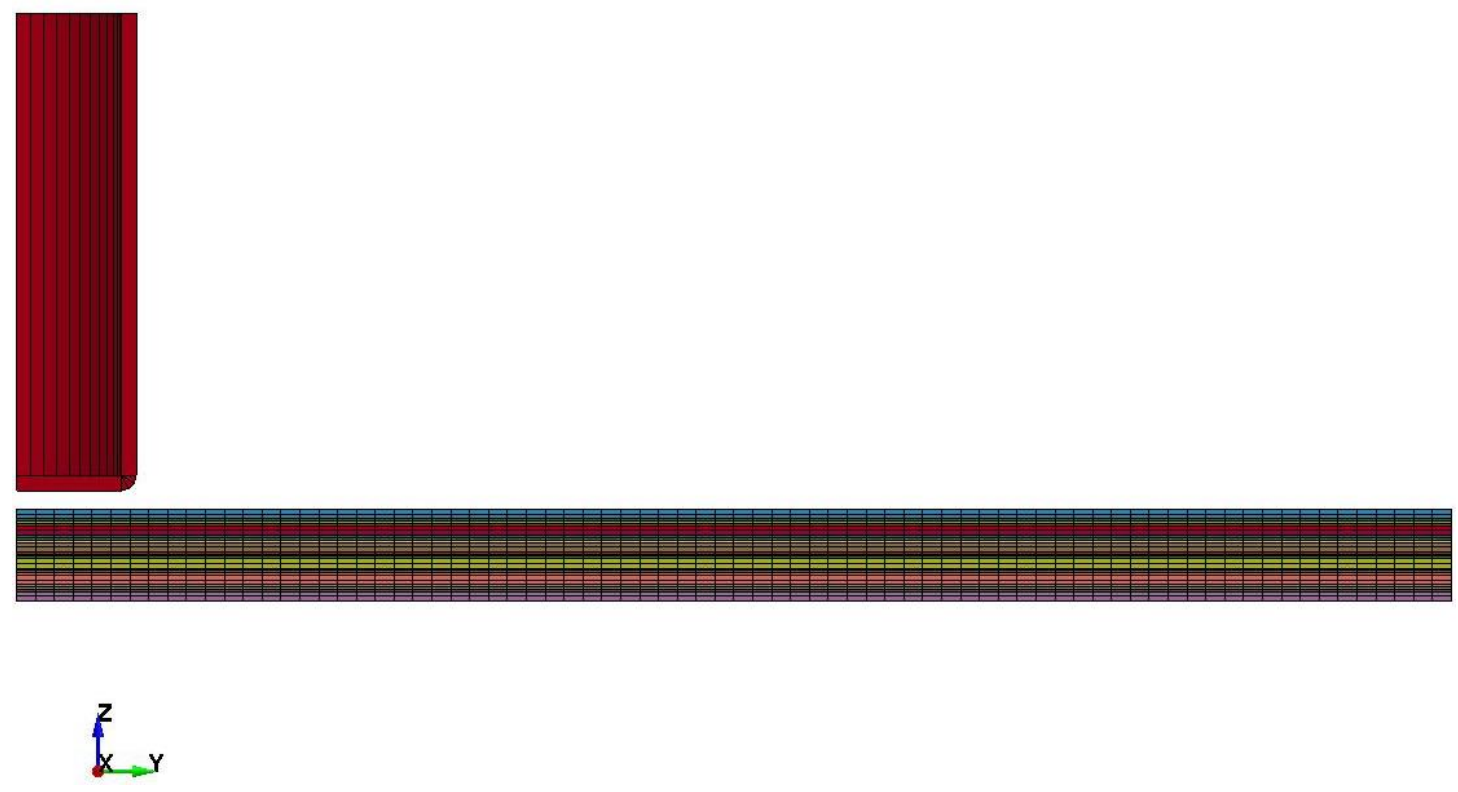

Fig. 1. Side view of the finite element mesh of a GLARE 4A-6/5-0.5 fiber-metal laminate along with the projectile.

\section{Results and discussion}

We apply the described FEM procedure to GLARE 4A-2/1-0.5, GLARE 4A-3/2-0.5, GLARE 4A-4/3-0.5, GLARE 4A-5/4-0.5 and GLARE 4A-6/5-0.5 material grades. 
Each 2024-T3 aluminum layer of these grades has a thickness of $0.5 \mathrm{~mm}$. Each prepreg ply has a thickness of $0.125 \mathrm{~mm}$ and consists of S2-glass UD fiber prepregs. The material properties of 2024-T3 aluminum and S2-glass fiber reinforced epoxy layers can be found in references [1,3]. Each intermediate composite laminate has thickness equal to $0.375 \mathrm{~mm}$ and layup: $0^{\circ}$ glass $/ 90^{\circ}$ glass $/ 0^{0}$ glass. For example, GLARE 4A-3/2-0.5 fiber-metal laminate consists of the following layup: $\left[2024-\mathrm{T} 3 / 0^{0}\right.$ glass $/ 90^{\circ}$ glass $/ 0^{0}$ glass $/ 2024-\mathrm{T} 3$ $/ 0^{0}$ glass $/ 90^{\circ}$ glass $/ 0^{0}$ glass $\left./ 2024-\mathrm{T} 3\right]$.

In Figure 2 the calculated 5 ballistic limits are compared. The corresponding 5 ballistic energies of the projectile are: 123.1, 156.8, 201.7, 252.3 and $274.1 \mathrm{~J}$. It can be seen from Figure 2 that the ballistic limit of GLARE 4A fiber-metal laminates can be increased significantly as the number of aluminum and glass-epoxy layers increases and the panel's layup becomes more complicated.

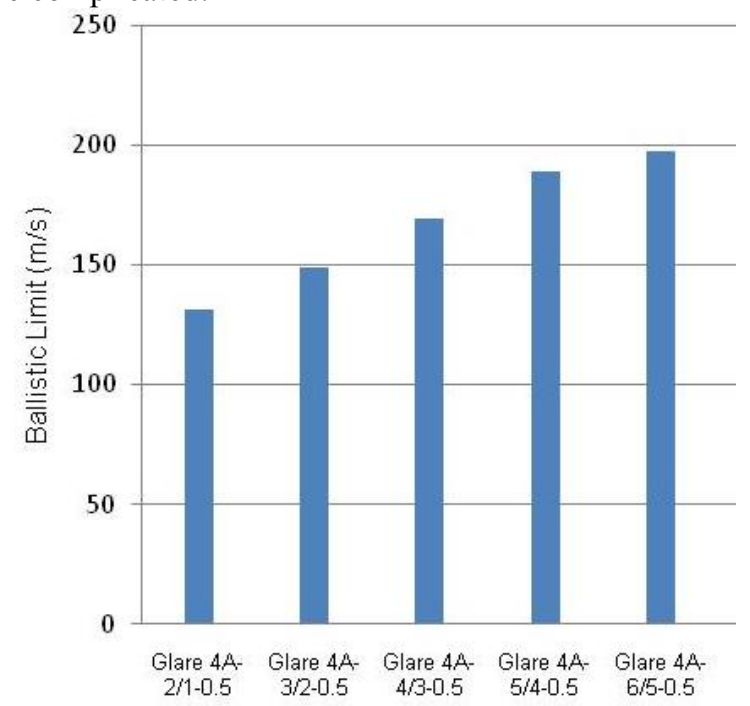

Fig. 2. Ballistic limits of GLARE 4A fiber-metal laminates.

In Figure 3 the absorbed impact energy time histories, obtained using the method described in [2], corresponding to the examined GLARE 4A panels are depicted. It can be observed from Figure 3 that there is a sudden increase of the absorbed impact energy at the beginning of the five ballistic impact phenomena. This increase is higher as the number of layers in the laminate increases. As discussed in the following paragraphs of this article, there is initially a sudden increase of the impact loading which is applied on the projectile due to its contact interaction with the target. This highly increased impact load is applied for a very short time interval in comparison with the duration of the ballistic impact event and yields a sudden drop of the projectile's kinetic energy which is reflected to the corresponding increase of the absorbed impact energy.

After the initial sudden absorbed impact energy variation the absorbed impact energy continues to increase with approximately constant rate which is lower in comparison with the beginning of impact. The major part of the initial impact energy is absorbed at this stage and is consumed to deform, cause damage and eventually perforate the panels. Then, at time $\mathrm{t} \approx 0.1 \mathrm{~ms}$, each curve of Figure 3 becomes gradually parallel to the horizontal axis as the striker perforates the targets and the rate of absorbed energy becomes gradually equal to zero due to the loss of contact between the colliding bodies, as the target is perforated. It is interesting to note that after the beginning of impact, the absorbed energy curves of Figure 3 are approximately parallel to each other. According to Figure 3, the final absorbed impact energy level increases for increasing number of layers in the GLARE 4A laminates. 


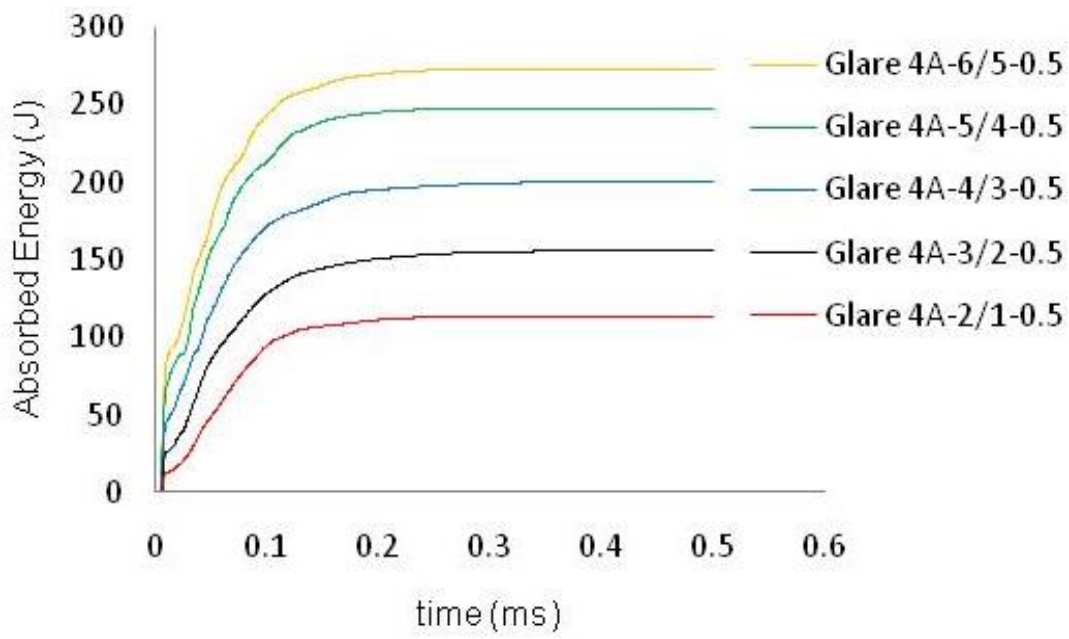

Fig. 3. Absorbed impact energy time history curves of GLARE 4A fiber-metal laminates subjected to ballistic impact by a cylindrical projectile.

In Figure 4 the impact load time histories of the considered GLARE 4A panels are depicted. We calculate each time history curve of impact load using the relation $F=m \gamma$, from the corresponding time history of the projectile's acceleration, which is obtained with our FEM analysis results. At time $\mathrm{t} \approx 0.3 \mathrm{~ms}$, all panels have been perforated and the perforation is reflected to the corresponding practically zero value of the impact load in Figure 4. The aforementioned peak impact load at the beginning of the ballistic impacts is shown in Figure 4. Significant sudden contact forces at the beginning of ballistic impact events, concerning target plates perforated by free-flying projectiles have also been found in the numerical simulations of references $[3,6,7]$. These contact forces are caused by the local indentation of the panel under the projectile during the beginning of impact. As soon as the local indentation stage is completed, the impact load drops suddenly, at time $t \approx$ $0.006 \mathrm{~ms}$, and the extended panel deformation stage, beyond the projectile's impacting face area, follows.

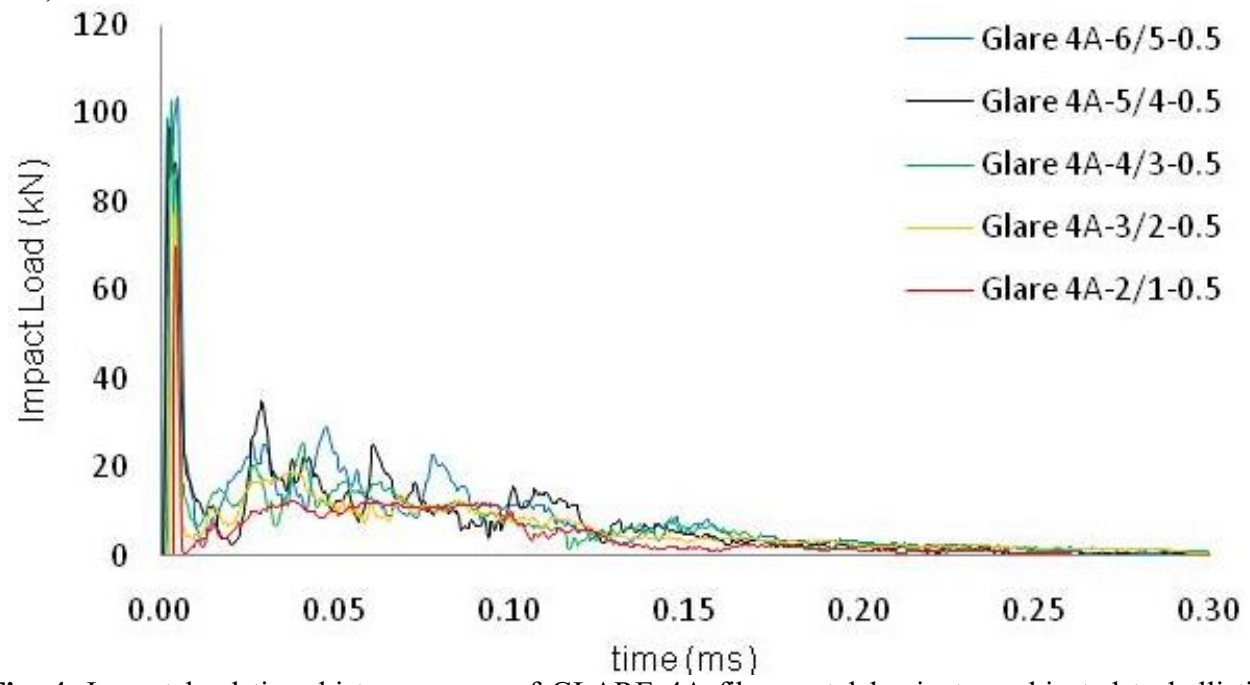

Fig. 4. Impact load time history curves of GLARE 4A fiber-metal laminates subjected to ballistic impact by a cylindrical projectile. 
The area under the impact load time history curve is equal to the impulse applied to the projectile during the collision of the two bodies. It can be observed from Figure 4, and more clear from Figure 5, that the impulse at the beginning of impact increases for increasing number of layers in the GLARE 4A laminates. This finding is reasonable, since both the stiffness of the target and the initial momentum of the projectile increase as the number of layers becomes larger and the associated ballistic limit is raised. Consequently, from the linear impulse-momentum law, the striker's momentum reduction becomes greater as the number of layers increases. Given that the projectile's mass is constant, the projectile's velocity reduction becomes greater as well. This is why initially there is a sudden increase of the absorbed impact energy, which becomes higher as the number of layers in the laminate increases, as discussed previously in association with Figure 3.

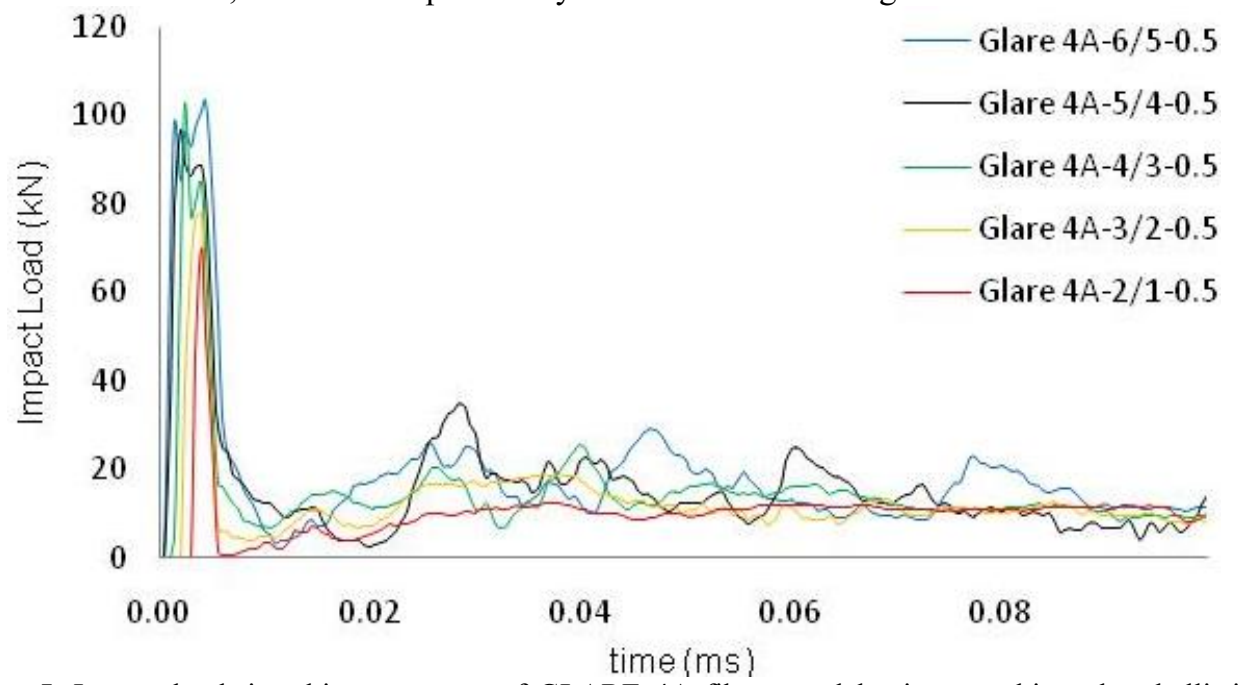

Fig. 5. Impact load time history curves of GLARE 4A fiber-metal laminates subjected to ballistic impact by a cylindrical projectile (part of the impact duration).

As shown in Figure 4, after the sudden drop of impact loading at the beginning of the events, the impact load increases in all cases due to the panel's resistance as it is forced to deform by the projectile, up to a local maximum impact load value. Then, until the perforation of the panels, the impact load time histories demonstrate noisy variations. The range of variations is smaller in the cases of GLARE 4A-2/1-0.5 and GLARE 4A-3/2-0.5. Significant sudden reductions of the impact load at this stage indicate further internal damages in the laminates caused by the striker.

In Figure 6 the impact load versus the projectile's displacement curves of the considered panels are depicted. It is noted that the projectile is initially located at a distance of $1 \mathrm{~mm}$ from the panels. As illustrated from Figure 6, all GLARE 4A panels have been perforated for a projectile's displacement equal to $21 \mathrm{~mm}$. The area under the impact loaddisplacement curve is equal to the work done by the contact force during the collision of the two bodies. It can be observed from Figure 6 that the work done at the beginning of impact increases for increasing number of layers in the GLARE 4A laminates. This finding is reasonable since, as discussed previously, the suddenly absorbed impact energy becomes higher as the number of the laminate layers increases. Thus, the associated reduction of the projectile's kinetic energy becomes higher as well. But according to the work-kinetic energy theorem, the variation of the striker's kinetic energy is equal to the work done by the impact load. This is why this work increases for increasing number of layers, as shown in Figure 6. 


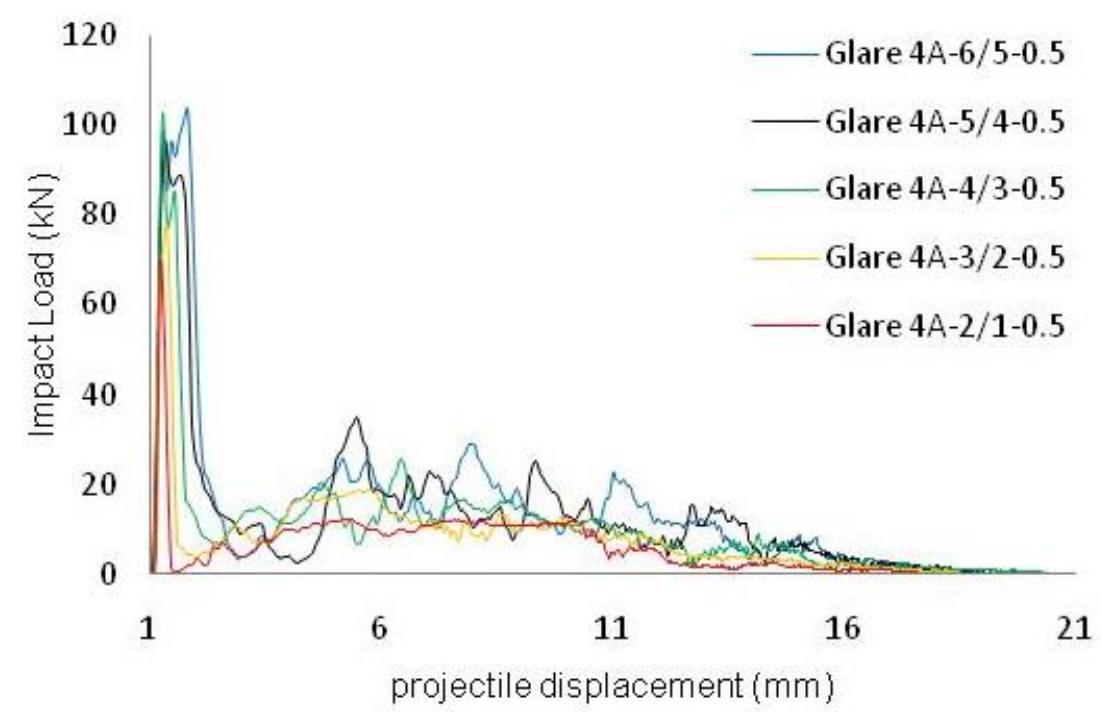

Fig. 6. Impact load versus striker's displacement curves of GLARE 4A fiber-metal laminates subjected to ballistic impact by a cylindrical projectile.

Using contours of the Von Mises stresses in the metal layers, the localized loading of the panel around the projectile at the beginning of the impact phenomenon is revealed. According to the calculations, the maximum effective stresses are above the yield stress of aluminum layers. Consequently, plasticity of the metal layers is an existing impact energy absorption mechanism from the beginning of the event.

In Figure 7 a 3D displacement contour of the projectile and a GLARE 4A panel is shown, at the end of the target's perforation. For this figure we have used LS-DYNA reflect model command. The figure corresponds to a perforated GLARE 4A-4/3-0.5 panel subjected to normal impact by the cylindrical projectile with the ballistic limit velocity. The displacement contour shows the nodal displacement vector in $\mathrm{m}$. A similar figure at the beginning of impact illustrates the detachment of the top aluminum layer around the boundary of the projectile due to shear failure mechanism. This failure mode is termed plugging and is associated with sub-ordnance impact of blunt projectiles on monolithic plates consisting of metals such as steel and aluminum [8]. The delamination of glassepoxy layers, a well known impact damage mechanism that occurs in composite materials and fiber-metal laminates [1], is also present at the beginning of the event. Figure 7 illustrates the projectile penetrating the perforated panel. The detached fragments of the panel can be observed under the projectile. The petaling of aluminum layers can also be observed around the projectile. Petaling deformations of aluminum in fiber-metal laminated targets have been observed experimentally in reference [1]. The debonding between aluminum and glass-epoxy layers is also indicated in the same figure. Extensive delamination/debonding damage among the layers of the laminate occurs as depicted in the figure. Another impact damage shown in Figure 7, is the failure of glass-epoxy layers, which has also been observed experimentally in ballistic impacts of fiber-metal laminates [1]. The simultaneous existence of the aforementioned impact damage mechanisms is unique in fiber-metal laminates. Each of the mechanisms absorbs a part of the initial impact energy and contributes to their high ballistic impact resistance. 


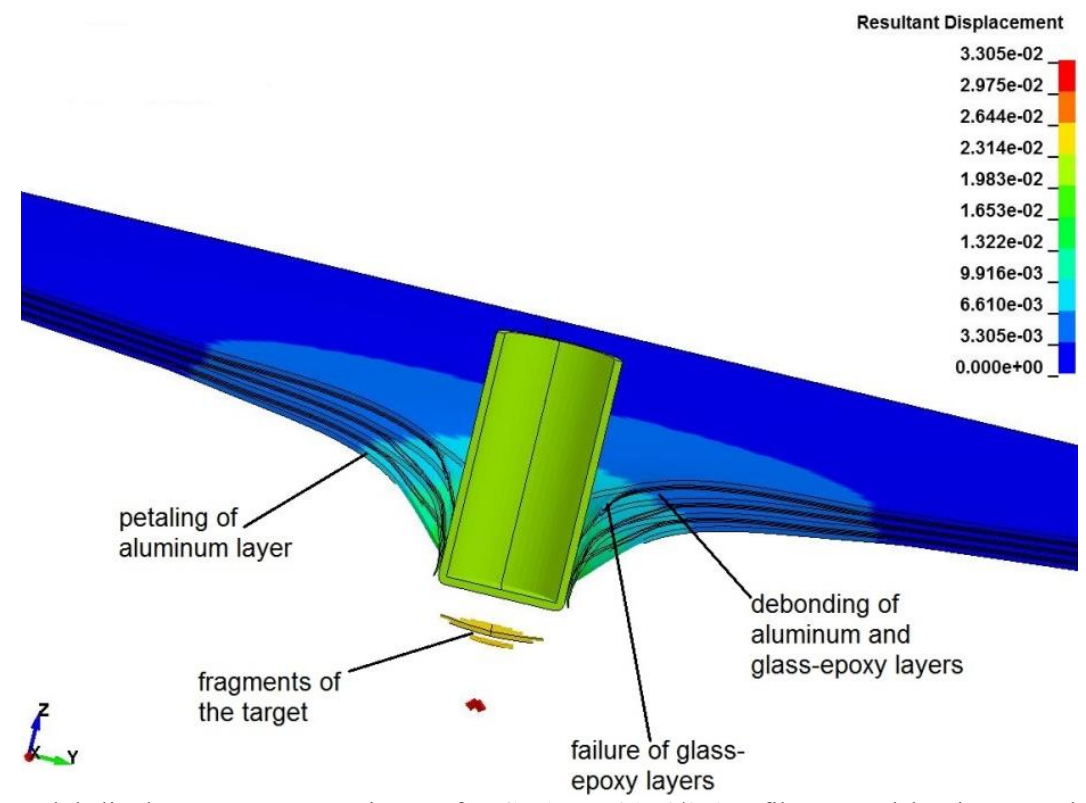

Fig. 7. Nodal displacement vectors in $m$ of a GLARE 4A-4/3-0.5 fiber-metal laminate at the end of the target's perforation by a cylindrical projectile

\section{Conclusions}

This article deals with the transient response of square clamped GLARE 4A fiber-metal laminates subjected to central normal ballistic impact by a rigid flat-faced cylindrical projectile. ANSYS LS-DYNA software is employed in order to simulate and study the ballistic impact phenomenon.

It is found that the ballistic limit of the GLARE 4A panels increases as their thickness becomes higher.

The maximum impact load is applied at the beginning of ballistic impacts, during the initial local indentation of the panels under the projectile. It is substantially higher than the following peak values of the impact force time history.

At the beginning of ballistic impacts, the impulse of the collision increases as the thickness of the panels is increased. The work done by the impact load during the local indentation stage is also an increasing function of the panels' thickness.

\section{References}

1. M.S. Hoo Fatt, C. Lin, D.M. Revilock jr, et al, Comp. Struct. 61, 73 (2003)

2. G.S.E. Bikakis, C.D. Dimou, E.P. Sideridis, Aerosp. Sci. Technol. 69, 201 (2017)

3. A.S. Yaghoubi, B. Liaw, J. Comp. Mater. 48, 2363 (2014)

4. LS-DYNA, Keyword user's manual (Livermore Software Technology Corporation, 2014)

5. LS-DYNA Support, http://www.dynasupport.com

6. B.A. Gama, J.W. Gillespie jr, Impact Eng. 38, 181 (2011)

7. T. Borvik, O.S. Hopperstad, T. Berstad, et al, Impact Eng. 27, 37 (2002)

8. R.S.J. Corran, P.J. Shadbolt, C. Ruiz, Impact Eng. 1, 3 (1983) 